\title{
On Achievable Rates of Interference and Cognitive Channels with a Relay
}

\author{
Marwen Zorgui ${ }^{1}$, Zouheir Rezki ${ }^{1}$ and Mohamed-Slim Alouini ${ }^{1}$ \\ ${ }^{1}$ Computer, Electrical, and Mathematical Sciences and Engineering (CEMSE) Division \\ King Abdullah University of Science and Technology (KAUST), Thuwal, Saudi Arabia \\ \{marwen.zorgui,zouheir.rezki,slim.alouini\}@kaust.edu.sa
}

\begin{abstract}
We consider a two-user interference channel assisted by a relay. Treating interference as noise at the receivers, and adopting an amplify and forward (AF) strategy at the relay, we derive achievable rates of both users, for given powers. Next, we solve the optimal power allocation problem maximizing the weighted sum rate of both users with and without relay power optimization. In particular, we propose a simple iterative line search algorithm solving the joint optimization problem over the three transmit powers and show that optimizing the relay power enhances the performance of the system. Then, considering the first user as a primary user, we determine the maximum instantaneous rate that the secondary user can achieve subject to an outage constraint with respect to the primary user and a peak power constraint. We show that, likewise the first part, jointly optimizing the secondary user and the relay transmit powers enhances the secondary user performance.
\end{abstract}

Index Terms-interference, relay, outage, power allocation.

\section{INTRODUCTION}

Cooperative communication have recently stirred a lot of research activities thanks to its promising applications. The underlying idea is that some mobile nodes in the network help each other communicate by acting as relays that forward the data from a source node to a destination node. This kind of cooperation has been shown to not only combat channel impairments and limitations, such as fading, shadowing and limited spectrum, but also to extend the network coverage. Based on the forwarding strategy and the processing carried out at the relay node, several relaying protocols have been proposed in the literature in order to improve the capacity and/or expand the coverage of wireless networks [1] [2] [3] [4]. The power allocated to the relay plays a role in the overall performance of the system. Optimal power allocation in amplify and forward (AF) networks has been investigated in [5] [6] [7]. However, most of these work focus on the single user scenario. Recently, there has been a growing interest in characterizing the potential advantages cooperation can provide in interference-limited systems, as opposed to the point-to-point scenarios [8] [9]. In [10], the authors studied the interference channel with cognitive relay (IFC-CR). The IFC$\mathrm{CR}$ comprises two independent source-destination pairs whose communication are aided by an additional node, refereed to as a cognitive relay (CR), who is assumed to possess a priori knowledge of both sources' messages. The authors derived outer and inner bounds on the capacity region of the general memoryless IFC-CR that are shown to be tight for certain classes of channels. Unfortunately, a general approach for dealing with interference in general is still unknown. The

The work of Z. Rezki and M. -S. Alouini was supported by the Qatar National Research Fund (a member of Qatar Foundation) under NPRP Grant NPRP 5-250-2-087. The statements made herein are solely the responsibility of the authors. capacity region of the interference channel is known for the case where both receivers experience strong interference [11], as in this case, the interference level is strong enough so that receivers can decode the interference first then decode their messages. Inspired by that, the authors in [12] introduced the idea of interference relaying, known also as interference forwarding (IF). IF aims at driving an interfered receiver into the very strong interference regime, which enables him to decode the impairment without rate penalty. In this regard, the authors in [13] investigated the effect of IF in the cognitive interference relay channel (CIRC), in which one of the transmitters (primary user) has a priori knowledge of the other transmitter's message (cognitive user). Paper [13] characterizes the capacity regions of two scenarios in case of strong interference. First scenario is when the interference forwarded by the relay consists of the primary user's signal and the second scenario corresponds to the case where the interference forwarded is the cognitive user's signal. It is shown that the capacity region in the second scenario is larger, i.e., when the relay does not help the intended (primary) receiver. In [14], the authors consider multi-level amplify-and forward relay networks with no direct link between the information sources and the destinations. Under full cooperation and full channel state information (CSI) at the destinations and no cooperation and no CSI at the sources and relay terminals, the ergodic capacity is determined and an asymptotic expression is derived based on the Stieltjes transform. In [15], the authors considered a multiple input multiple output (MIMO) cognitive radio coexisting with a MIMO primary radio under a novel primary user rate constraint, and they proposed an iterative algorithm to maximize the cognitive user transmission rate.

In this paper, we consider a two-user channel assisted by a relay. Both transmitters and receivers do not cooperate and each receiver does not "see" directly the transmitted symbol from the other transmitter, but only does so through the relay. These assumptions are reasonable when strong shadowing affects the links from both transmitters to the non-intended receivers in a wireless communication environment. The relay receives signals from both transmitters and is seen by both receivers as well. We study the described system in the quasi-static fading where all channels are fixed. Moreover, we assume that all terminals are equipped with a single antenna. Furthermore, our focus is on AF relaying strategy, although more sophisticated relaying strategies may provide some performance gain.

The organization of this paper is as follows. In Section II, the system model and the corresponding assumptions are described and achievable rates are derived. In Section III, we solve the optimal power allocation problem maximizing 


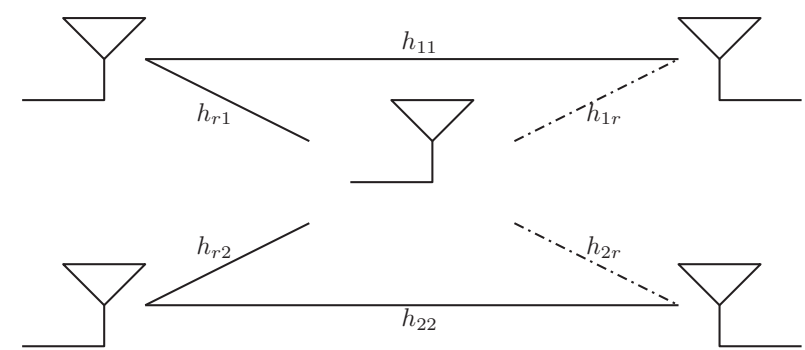

Fig. 1. System model of a two-user system assisted with a relay

the total throughput of the system, with and without relay power optimization. In Section IV, we solve the problem of maximizing the instantaneous secondary user achievable rate subject to outage and peak power constraints. Conclusions are presented in Section V. We support our analysis with numerical results.

\section{SYSTEM MODEL AND ACHIEVABLE RATES}

As illustrated in Fig. 1, we consider a two-user interference channel assisted by a relay. We assume that the relay and each receiver have full knowledge of their incoming channel coefficients. Moreover, the relay forwards his channel coefficients $\left(h_{r 1}\right.$ and $\left.h_{r 2}\right)$ to both transmitters while each receiver transmits his channel coefficients $\left(h_{i r}\right.$ and $\left.h_{i i}, i \in\{1,2\}\right)$ to his corresponding transmitter only. In the first time slot, each user transmits a symbol from his codeword and we have the following equations at the corresponding receivers and the relay terminal (corresponding to solid lines in Fig. 1)

$$
\begin{aligned}
y_{1,1} & =h_{11} x_{1}+n_{1,1} \\
y_{2,1} & =h_{22} x_{2}+n_{2,1} \\
y_{r} & =h_{r 1} x_{1}+h_{r 2} x_{2}+n_{r},
\end{aligned}
$$

where $h_{i j}$ is the channel coefficient between receiver $i$ and transmitter $j$ for $i, j \in\{1,2, r\}$ and $n_{1,1}, n_{2,1}$ and $n_{r}$ correspond to the additive Gaussian noise at the first receiver, the second receiver and the relay, respectively. Without loss of generality, the noises are assumed to be distributed according to a zero mean unit variance Gaussian distribution (denoted $\sim \mathcal{C N}(0,1)$ ). In the second time slot, the relay amplifies his received signal and forwards the signal $x_{r}=G y_{r}$. The received signals at both receivers can be expressed as (corresponding to dash-dotted lines in Fig. 1)

$$
\begin{aligned}
y_{1,2} & =h_{1 r} x_{r}+n_{1,2} \\
& =h_{1 r} G\left(h_{r 1} x_{1}+h_{r 2} x_{2}+n_{r}\right)+n_{1,2} \\
& =G h_{1 r} h_{r 1} x_{1}+\left(G h_{r 2} h_{1 r} x_{2}+G h_{1 r} n_{r}+n_{1,2}\right) \\
y_{2,2} & =G h_{2 r} h_{r 2} x_{2}+\left(G h_{r 1} h_{2 r} x_{1}+G h_{2 r} n_{r}+n_{2,2}\right) .
\end{aligned}
$$

Similarly, we have $n_{1,2}, n_{2,2} \sim \mathcal{C N}(0,1)$. The relay is subject to an average power constraint given by

$$
\mathbb{E}\left(\left|x_{r}\right|^{2}\right) \leq P_{r} .
$$

We define $\gamma_{i j} \triangleq\left|h_{i j}\right|^{2}$ for $i \in\{1,2, r\}$ and $j \in\{1,2, r\}$. Then, (6) translates into a constraint on the multiplicative factor $\mathrm{G}$, expressed as

$$
G^{2} \leq \frac{P_{r}}{\gamma_{r 1} P_{1}+\gamma_{r 2} P_{2}+1} .
$$

We assume that each transmitter is employing a Gaussian codebook. Moreover, the interference is treated as noise. Hence, from the perspective of receiver 1, this results in an equivalent noise with variance $\sigma_{1,2}^{2}=G^{2} \gamma_{r 2} \gamma_{1 r} P_{2}+G^{2} \gamma_{1 r}+$ 1. Without loss of generality, the observation at the first receiver in the second time slot can be expressed as

$$
y_{1,2}^{e q}=\frac{G}{\sigma_{1,2}} h_{1 r} h_{r 1} x_{1}+n_{1,2}^{e q},
$$

such that $n_{1,2}^{e q} \sim \mathcal{C N}\{0,1\}$. The equivalent observations at the first receiver over the two time slots can be expressed as

$$
\mathbf{y}_{1}=\left(\begin{array}{c}
h_{11} \\
\frac{G}{\sigma_{1,2}} h_{1 r} h_{r 1}
\end{array}\right) x_{1}+\left(\begin{array}{c}
n_{1,1} \\
n_{1,2}^{e q}
\end{array}\right)=\mathbf{h}_{1} x_{1}+\mathbf{n}_{1},
$$

such that $\mathbf{n}_{1} \sim \mathcal{C N}\{0, \mathbf{I}\}$. The achievable rate of transmitter 1 is given by [16]

$$
\begin{aligned}
R_{1} & =\frac{1}{2} \mathbb{I}\left(x_{1} ; \mathbf{y}_{1}\right) \\
& =\frac{1}{2} \log \left(1+P_{1}\left\|\mathbf{h}_{1}\right\|^{2}\right) \\
& =\frac{1}{2} \log \left(1+P_{1} \gamma_{11}+\frac{P_{1} G^{2} \gamma_{1 r} \gamma_{r 1}}{G^{2} \gamma_{r 2} \gamma_{1 r} P_{2}+G^{2} \gamma_{1 r}+1}\right) .
\end{aligned}
$$

Similarly, the achievable rate of user 2 is given by

$$
R_{2}=\frac{1}{2} \log \left(1+P_{2} \gamma_{22}+\frac{P_{2} G^{2} \gamma_{2 r} \gamma_{r 2}}{G^{2} \gamma_{r 1} \gamma_{2 r} P_{2}+G^{2} \gamma_{2 r}+1}\right) .
$$

From (10) and (11), it is clear that it is optimal to choose the maximum value for $G^{2}$ in order to maximize both achievable rates. In the sequel, $\mathrm{G}$ is chosen to satisfy $G^{2}=$ $\frac{P_{r}}{\gamma_{r 1} P_{1}+\gamma_{r 2} P_{2}+1}$. We define $\eta_{i j} \triangleq \gamma_{i j} P_{j}$ for $i \in\{1,2, r\}$ and $j \in\{1,2, r\}$. Substituting $G^{2}$ by its maximum value, we obtain after simplification

$$
\begin{aligned}
& R_{1} \\
& =\frac{1}{2} \log \left(1+P_{1} \gamma_{11}+\frac{\gamma_{1 r} P_{r} \gamma_{r 1} P_{1}}{\gamma_{r 2} P_{2} \gamma_{1 r} P_{r}+\gamma_{1 r} P_{r}+\gamma_{r 1} P_{1}+\gamma_{r 2} P_{2}+1}\right) \\
& =\frac{1}{2} \log \left(1+\eta_{11}+\frac{\eta_{1 r} \eta_{r 1}}{\eta_{r 2} \eta_{1 r}+\eta_{1 r}+\eta_{r 1}+\eta_{r 2}+1}\right) \\
& =\frac{1}{2} \log \left(1+\eta_{11}+\frac{\eta_{1 r} \eta_{r 1} /\left(1+\eta_{r 2}\right)}{1+\eta_{1 r}+\eta_{r 1} /\left(1+\eta_{r 2}\right)}\right) .
\end{aligned}
$$

Similarly, The achievable rate of transmitter 2 is written as $R_{2}$

$$
\begin{aligned}
& =\frac{1}{2} \log \left(1+P_{2} \gamma_{22}+\frac{\gamma_{2 r} P_{r} \gamma_{r 2} P_{2}}{\gamma_{r 1} P_{1} \gamma_{2 r} P_{r}+\gamma_{2 r} P_{r}+\gamma_{r 2} P_{2}+\gamma_{r 1} P_{1}+1}\right) \\
& =\frac{1}{2} \log \left(1+\eta_{22}+\frac{\eta_{2 r} \eta_{r 2} /\left(1+\eta_{r 1}\right)}{1+\eta_{2 r}+\eta_{r 2} /\left(1+\eta_{r 1}\right)}\right) .
\end{aligned}
$$

Next, we maximize the achievable rate-region of the interference channel with a relay subject to power constraints in Section III. Then, in Section IV, we consider a cognitive channel and we use (14) and (16) in order to maximize the achievable rate of user 2 subject to a quality of service constraint imposed by user 1 .

\section{TOTAL THROUGHPUT MAXIMIZATION}

In this section, we seek to maximize the achievable rateregion under a sum power constraint. Note that this problem requires a global knowledge of all channel gains. We first 
consider that the relay power is fixed. Then, we optimize over all involved powers.

\section{A. maximizing the throughput with fixed relay power}

Our optimization problem is written as

$$
\begin{array}{cl}
\underset{P_{1}, P_{2}}{\operatorname{maximize}} & w R_{1}\left(P_{1}, P_{2}\right)+(1-w) R_{2}\left(P_{1}, P_{2}\right) \\
\text { subject to } & P_{1}+P_{2} \leq \bar{P} \\
& 0 \leq P_{i} \text { for } i \in\{1,2\},
\end{array}
$$

where $w \in[0,1]$. Each value of $w$ gives a point on the boundary of the achievable rate-region. To solve (17), we make use of the following lemma.

Lemma 1. The optimal solution of (17) should consume all the available power, i.e., $P_{1}^{o p t}+P_{2}^{o p t}=\bar{P}$.

Proof: Suppose that $P_{1}^{o p t}+P_{2}^{o p t}=\bar{P}-\Delta P$ such that $\Delta P>0$. Let $\alpha=1+\frac{\Delta P}{P_{1}^{o p t}+P_{2}^{o p t}}$ and let $P_{1}=\alpha P_{1}^{o p t}, P_{2}=$ $\alpha P_{2}^{o p t}$. Clearly, $P_{1}+P_{2}=\bar{P}^{2}$. From (12) and (15), we can write

$$
\begin{aligned}
R_{1}\left(P_{1}, P_{2}\right) & =\frac{1}{2} \log \left(1+\alpha P_{1}^{o p t} \gamma_{11}\right. \\
& \left.+\frac{\gamma_{1 r} P_{r} \gamma_{r 1} P_{1}^{o p t}}{\gamma_{r 2} P_{2}^{o p t} \gamma_{1 r} P_{r}+\frac{\gamma_{1 r} P_{r}}{\alpha}+\gamma_{r 1} P_{1}^{o p t}+\gamma_{r 2} P_{2}^{o p t}+\frac{1}{\alpha}}\right) \\
R_{2}\left(P_{1}, P_{2}\right) & =\frac{1}{2} \log \left(1+\alpha P_{2}^{o p t} \gamma_{22}\right. \\
& \left.+\frac{\gamma_{2 r} P_{r} \gamma_{r 2} P_{2}^{o p t}}{\gamma_{r 1} P_{1}^{o p t} \gamma_{2 r} P_{r}+\frac{\gamma_{2 r} P_{r}}{\alpha}+\gamma_{r 2} P_{2}^{o p t}+\gamma_{r 1} P_{1}^{o p t}+\frac{1}{\alpha}}\right) .
\end{aligned}
$$

As $\alpha>1$, we have $R_{1}\left(P_{1}, P_{2}\right)>R_{1}\left(P_{1}^{o p t}, P_{2}^{o p t}\right)$ and $R_{2}\left(P_{1}, P_{2}\right)>R_{2}\left(P_{1}^{o p t}, P_{2}^{o p t}\right)$. Hence, $\left(P_{1}, P_{2}\right)$ satisfies the power constraint and achieves a strictly higher sum rate. This contradicts the assumption that $P_{1}^{o p t}+P_{2}^{o p t}<\bar{P}$.

Therefore, the optimization problem in (17) can be expressed as one variable (for example $P_{1}$ ) optimization problem by setting $P_{2}=\bar{P}-P_{1}$ and replacing it in the objective function. We write then

$$
\begin{array}{ll}
\underset{P_{1}}{\operatorname{maximize}} & w R_{1}\left(P_{1}, \bar{P}-P_{1}\right)+(1-w) R_{2}\left(P_{1}, \bar{P}-P_{1}\right) \\
\text { subject to } & 0 \leq P_{1} \leq \bar{P},
\end{array}
$$

The objective function (20) is not guaranteed to be concave. To obtain the optimal solution, we should search over the boundary points $\left(P_{1}=0, P_{1}=\bar{P}\right)$ and the extreme points corresponding to $\frac{\partial\left(w R_{1}\left(P_{1}, \bar{P}-P_{1}\right)+(1-w) R_{2}\left(P_{1}, \bar{P}-P_{1}\right)\right)}{\partial P_{1}}=0$.

\section{B. maximizing the throughput over all power variables}

Optimizing over the relay power should enhance the system performance. The problem is now formulated as

$$
\begin{array}{cl}
\underset{P_{1}, P_{2}, P_{r}}{\operatorname{maximize}} & w R_{1}\left(P_{1}, P_{2}, P_{r}\right)+(1-w) R_{2}\left(P_{1}, P_{2}, P_{r}\right) \\
\text { subject to } & P_{1}+P_{2}+P_{r} \leq \bar{P} \\
& 0 \leq P_{i} \text { for } i \in\{1,2, r\} .
\end{array}
$$

Once again, we note that, by proceeding as in Lemma 1, one can check that at the optimal, we should use all the available power. Consequently, one can eliminate one variable (say $P_{r}$ ) and solve an optimization problem over two variables. The problem is formulated then as

$$
\begin{array}{cl}
\underset{P_{1}, P_{2}}{\operatorname{maximize}} & w R_{1}\left(P_{1}, P_{2}, \bar{P}-P_{1}-P_{2}\right) \\
& +(1-w) R_{2}\left(P_{1}, P_{2}, \bar{P}-P_{1}-P_{2}\right) \\
\text { subject to } & P_{1}+P_{2} \leq \bar{P} \\
& 0 \leq P_{i} \text { for } i \in\{1,2\} .
\end{array}
$$

Note that (22) is not a convex optimization problem. To solve it, one can perform an exhaustive search or one has to evaluate the objective function at all candidate points (corner points, relative maxima on the boundary and the interior of the domain). While the first method is costly, the second approach is cumbersome and tedious given the objective function expression. To overcome this difficulty, we propose an algorithm (see Algorithm 1) that is simple to implement in order to solve (21). The advantage of using Algorithm 1

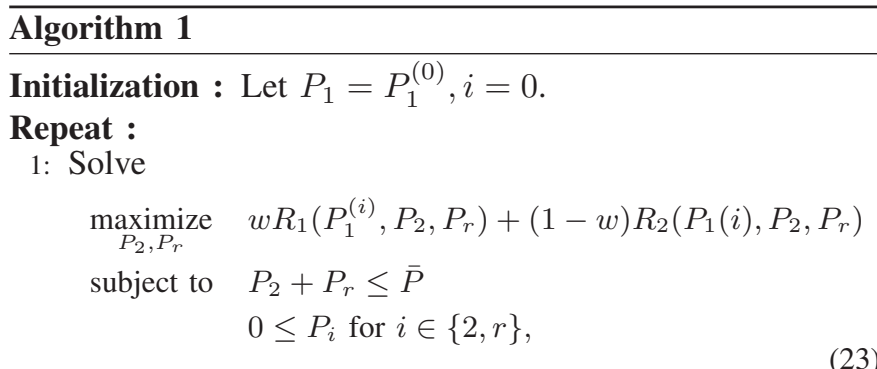

Denote the maximum in (23) by $f_{1}^{(i)}$ and let $P_{2}^{(i)} \leftarrow P_{2}^{o p t}$. 2: Solve

$$
\begin{array}{cl}
\underset{P_{1}, P_{r}}{\operatorname{maximize}} & w R_{1}\left(P_{1}, P_{2}^{(i)}, P_{r}\right)+(1-w) R_{2}\left(P_{1}, P_{2}^{(i)}, P_{r}\right) \\
\text { subject to } & P_{1}+P_{r} \leq \bar{P} \\
& 0 \leq P_{i} \text { for } i \in\{1, r\},
\end{array}
$$

Denote the maximum in (24) by $f_{2}^{(i)}$ and let $P_{1}^{(i+1)} \leftarrow$ $P_{1}^{o p t}, \Delta^{(i)}=\left|f 1^{(i)}-f 2^{(i)}\right|, i \leftarrow i+1$.

Until : $\Delta^{(i-1)} \geq \delta$,

where $\delta$ is an arbitrary small positive constant.

is that the successive optimization problems solved in steps 1 and 2 can be turned into a one variable optimization problem. Indeed, consider for example the optimization problem in step 1. Since both instantaneous rates are increasing in $P_{r}$, then, for a given $\left(P_{2}, P_{r}\right)$ satisfying the constraints, by increasing $P_{r}$ while satisfying the sum power constraint, one can achieve higher rates $R_{1}$ and $R_{2}$ simultaneously. Thus, the inequality should be satisfied with equality at the optimal point. We note that while the proposed algorithm converges to the global solution in most scenarios, it can provide however, a local maximum, as illustrated in the following example.

Example 1. We consider the following setup: $\bar{P}=$ $20 \mathrm{~dB}, \gamma_{11}=0.0264, \gamma_{22}=0.0747, \gamma_{r 1}=1.4051, \gamma_{r 2}=$ $3.1994, \gamma_{1 r}=0.6651, \gamma_{2 r}=2.7702, w=0.5$, and $\delta=$ $10^{-10}$. In Fig. 2, we visualize the objective function in (22). For $P_{1}^{(0)}=\frac{\bar{P}}{2}$, the proposed algorithm provides a local maximum, given by the point $\left(P_{1}, P_{2}\right)=(42.2819,0)$ (shown with a box) whereas the maximum in the considered setup is 


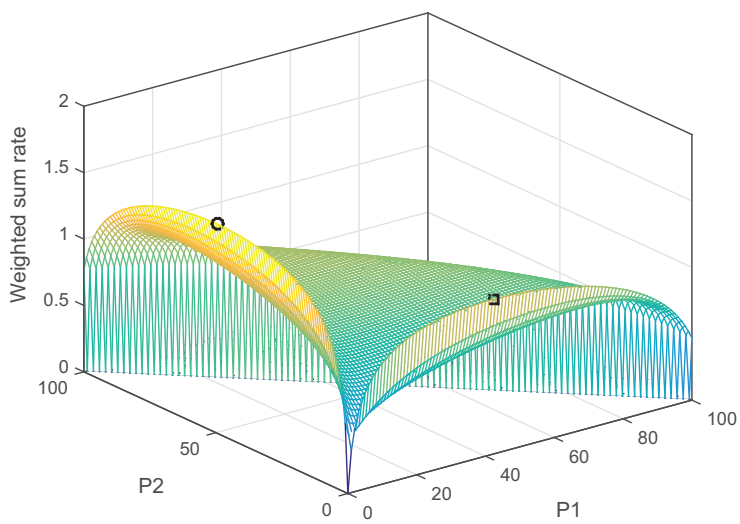

Fig. 2. Weighted sum rate over the feasible region

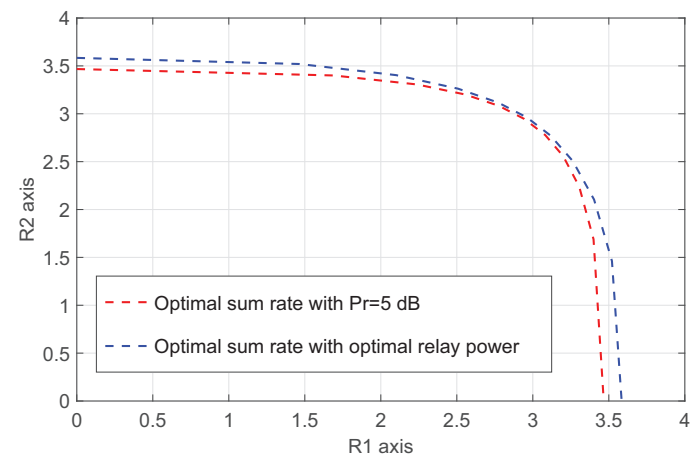

Fig. 3. Achievable rate region

given by $\left(P_{1}, P_{2}\right)=(0,49.5)$ (shown with a circle).

Example 2. We consider Rayleigh fading such that $\Omega_{i, j}=2$ for all $(i, j)$. The total power budget is set to $\bar{P}=20 \mathrm{~dB}$. We let the parameter $w$ sweep the axis $[0,1]$. We plot the achievability region resulting from the optimization problems (17) and (21). To solve (17), we assume a fixed relay power $P_{r}=5 \mathrm{~dB}$. Figures 3 and 4 are obtained by averaging $10^{5}$ simulations. Figure 3 shows clearly that optimizing the relay power enhances the overall throughput of the system. Figure 4 plots the relay power allocation versus $w$. The symmetry observed is expected given the model. More interestingly, the lowest relay power is obtained for a fair throughput objective function $(w=0.5)$. The relay power increases as the parameter $w$ becomes more biased in favor of any user.

\section{MAXIMIZING The SeCondary USER ACHIEVABLE RATE}

In this section, our objective is to maximize the secondary user achievable rate. The first transmitter is assumed to operate with an instantaneous peak power constraint. To maximize his achievable rate, transmitter 1 operates with full power $P_{1}$. On the other hand, we seek to maximize the instantaneous achievable rate of user 2 . Note that transmitter 2 is assumed to have knowledge only of $h_{22}, h_{2 r}, h_{r 1}$ and $h_{r 2}$, but he is not aware of the realizations of $h_{11}$ and $h_{1 r}$. However, the

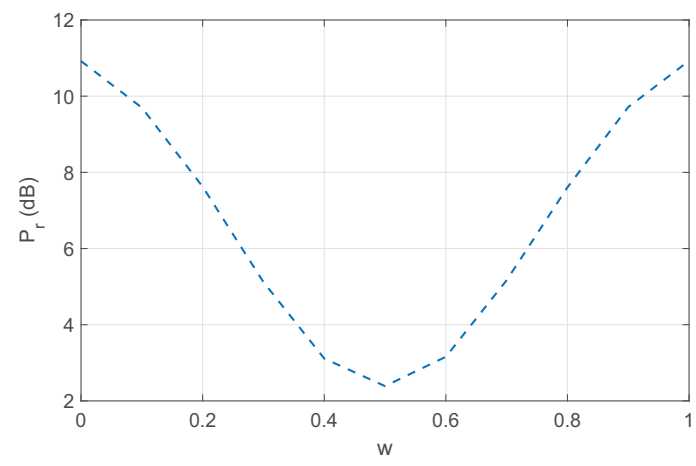

Fig. 4. Optimal relay power allocation

secondary transmitter is assumed to have statistical knowledge about the probability distributions of $h_{11}$ and $h_{1 r}$. Therefore, a power allocation should satisfy a quality of service constraint with respect to the primary user. For instance, we impose an outage probability constraint that the secondary user should satisfy. We first consider that the relay power is fixed.

A. maximizing the secondary rate with fixed relay power

Assuming that the relay power is fixed, we note from (15) that maximizing the secondary rate is equivalent to maximizing his transmit power $P_{2}$. Thus, we write

$$
\begin{array}{cl}
\underset{P_{2}}{\operatorname{maximize}} & P_{2} \\
\text { subject to } & 0 \leq P_{2} \leq \bar{P}_{2}, \\
& \operatorname{Pr}\left(R_{1}<R_{t} \mid \gamma_{22}, \gamma_{2 r}, \gamma_{r 1}, \gamma_{r 2}\right) \leq \epsilon .
\end{array}
$$

The outage constraint can be expressed as

$$
\begin{aligned}
P_{\text {out }, 1} & =\operatorname{Pr}\left(R_{1}<R_{t} \mid \gamma_{22}, \gamma_{2 r}, \gamma_{r 1}, \gamma_{r 2}\right) \\
& =\operatorname{Pr}\left(\eta_{11}+\frac{\eta_{1 r} \eta_{r 1} /\left(1+\eta_{r 2}\right)}{1+\eta_{1 r}+\eta_{r 1} /\left(1+\eta_{r 2}\right)} \leq 2^{2 R_{t}}-1\right) \\
& =\operatorname{Pr}\left(\frac{\eta_{1 r} \eta_{r 1} /\left(1+\eta_{r 2}\right)}{\eta_{1 r}+1+\eta_{r 1} /\left(1+\eta_{r 2}\right)} \leq 2^{2 R_{t}}-1-\eta_{11}\right) .
\end{aligned}
$$

We note that $P_{\text {out }, 1}$ is continuous in $P_{2}$. Moreover, from (13), $R_{1}$ is strictly decreasing with respect to $P_{2}$, hence $P_{\text {out }, 1}$ is increasing with respect to $P_{2}$. Therefore, $P_{\text {out }, 1}$ is an invertible function with respect to $P_{2}$, and the outage constraint is equivalent to an upper bound constraint on $P_{2}$.

$$
P_{\text {out }, 1}\left(P_{2}\right) \leq \epsilon \Leftrightarrow P_{2} \leq P_{\text {out }, 1}^{-1}(\epsilon) .
$$

Details of computing $P_{\text {out }, 1}$ can be found in Appendix A. Note that for (27) to be feasible, we should have $\epsilon \geq \epsilon_{0}$ where $\epsilon_{0}=P_{\text {out }, 1}\left(P_{2}\right)_{\mid P_{2}=0}$. Otherwise, the problem is infeasible and we set $P_{2}=0$. The optimization problem (25) becomes

$$
\begin{array}{cl}
\underset{P_{2}}{\operatorname{maximize}} & P_{2} \\
\text { subject to } & 0 \leq P_{2} \leq \bar{P}_{2}, \\
& P_{2} \leq P_{\text {out }, 1}^{-1}(\epsilon) .
\end{array}
$$

The optimal solution is given by $P_{2}^{\text {opt }}=\min \left(\bar{P}_{2}, P_{\text {out }, 1}^{-1}(\epsilon)\right)$.

Example 3. We consider Rayleigh fading, i.e., channel coefficients $h_{i, j}$ are generated i.i.d. $h_{i, j} \sim \mathcal{C N}\left(0, \Omega_{i, j}\right)$. We set 


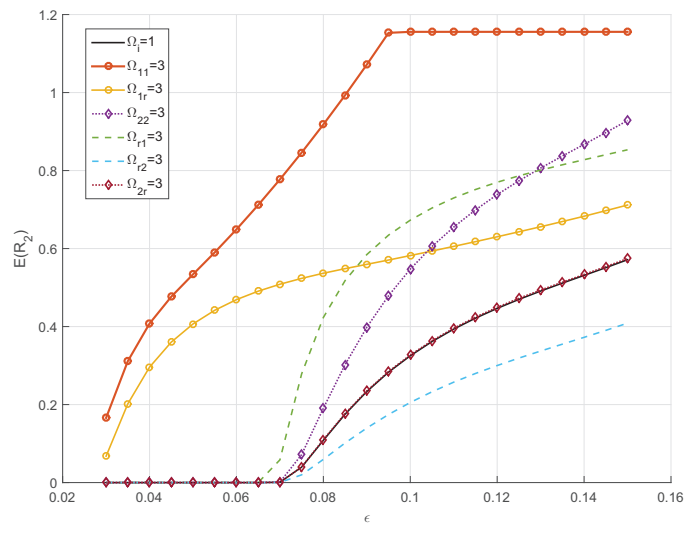

Fig. 5. Average achievable rate of User 2

$R_{t}=1 \mathrm{bit} / \mathrm{s} / \mathrm{Hz}, P 1=10 \mathrm{~dB}, \bar{P}_{2}=3 \mathrm{~dB}, P_{r}=7 \mathrm{~dB}$. In Fig. 5 , we plot the achievable rate of user 2 subject to the outage and peak power constraints for different values of outage upper bounds. We average the results over $10^{5}$ realizations. We first plot the system performance for the case when all channel link parameters are unity, i.e., $\Omega_{i j}=1$. We then increase one link parameter one at a time while maintaining the other links to unity and study the impact of each link on the system performance, on average. Increasing $\Omega_{r 2}$ decreases the achievable rate of user 2. This is explained by the fact that increasing the value of $\gamma_{r 2}$ increases the interference power at user 1 and hence increases the outage probability. This results in decreasing the optimal transmit power of user 2 , which dominates the positive impact of increasing $\gamma_{r 2}$ on the achievable rate $R_{2}$. As opposed to that, increasing the remaining links increases the achievable rate of user 2 , with a different impact of each link. The maximum beneficial impact is obtained by having a stronger link $\gamma_{11}$ as this link enhances the achievable rate of user 1 and decreases considerably the outage probability, allowing user 2 to transmit at a higher power level. While $\gamma_{22}$ and $\gamma_{2 r}$ do not affect the outage event, they contribute to the achievable rate $R_{2}$ with the impact of $\gamma_{22}$ dominating the impact of $\gamma_{2 r}$. Note that for the curve corresponding to $\Omega_{2 r}$, we experience a very small increase of $R_{2}$, a higher value of $\Omega_{2 r}$ is required to observe a considerable increase.

B. Power control at the secondary transmitter and the relay

In this subsection, we assume that the first transmitter is still operating with a fixed power $P_{1}$, while we now optimize the secondary user achievable rate jointly over both $P_{2}$ and the relay power $P_{r}$. The optimization problem becomes

$$
\begin{array}{cl}
\underset{P_{2}, P_{r}}{\operatorname{maximize}} & R_{2}\left(P_{2}, P_{r}\right) \\
\text { subject to } & 0 \leq P_{2}+P_{r} \leq \bar{P}, \\
& \operatorname{Pr}\left(R_{1}<R_{t} \mid \gamma_{22}, \gamma_{2 r}, \gamma_{r 1}, \gamma_{r 2}\right) \leq \epsilon .
\end{array}
$$

In order to solve (29), we note that the power constraint should be satisfied with equality. Otherwise, as both instantaneous rates are increasing in $P_{r}$, then, for a given $\left(P_{2}, P_{r}\right)$ satisfying the constraints in (29), by increasing $P_{r}$, one can satisfy the outage constraint and can achieve a higher rate $R_{2}$ simulta- neously. Henceforth, the inequality should be satisfied with equality at the optimal point. Thus, (29) can be written as a function of $P_{2}$ only as follows

$$
\begin{array}{cl}
\underset{P_{2}}{\operatorname{maximize}} & R_{2}\left(P_{2}, \bar{P}-P_{2}\right) \triangleq R_{2}^{e q}\left(P_{2}\right) \\
\text { subject to } & 0 \leq P_{2} \leq \bar{P}, \\
& \operatorname{Pr}\left(R_{1}\left(P_{2}, \bar{P}-P_{2}\right)<R_{t} \mid \gamma_{22}, \gamma_{2 r}, \gamma_{r 1}, \gamma_{r 2}\right) \leq \epsilon,
\end{array}
$$

such that the optimal relay power is given by $P_{r}^{o p t}=\bar{P}-P_{2}^{o p t}$ and $P_{2}^{o p t}$ is the solution to (30). From (36), the achievable rate $R_{1}$ is decreasing in $P_{2}$. Moreover, $R_{1}$ is increasing in $P_{r}$, hence it is decreasing in $\bar{P}-P_{2}$. Therefore, $R_{2}^{e q}\left(P_{2}\right)$ is decreasing in $P_{2}$. Given that, the outage constraint at user 1 is equivalent to an upper bound on $P_{2}$.

$$
P_{\text {out }, 1}\left(P_{2}, \bar{P}-P_{2}\right) \leq \epsilon \Leftrightarrow P_{2} \leq P_{\text {out }, 1}^{-1}(\epsilon) .
$$

Note that in order to compute $P_{\text {out }, 1}\left(P_{2}, \bar{P}-P_{2}\right)$, we start from (36) and evaluate the expression after replacing $P_{r}=\bar{P}-P_{2}$. Similarly, we note that for (31) to be feasible, we should have $\epsilon \geq \epsilon_{0}$ where $\epsilon_{0}=P_{\text {out }, 1}\left(P_{2}, P_{r}\right)_{\mid P_{2}=0, P_{r}=\bar{P}}$. Otherwise, we set $P_{2}=0$.

To solve (30), we first express $R_{2}$ as

$$
\begin{aligned}
& R_{2}^{e q}\left(P_{2}\right)=\frac{1}{2} \log \left(1+P_{2} \gamma_{22}\right. \\
& \left.+\frac{\gamma_{2 r}\left(\bar{P}-P_{2}\right) \gamma_{r 2} P_{2}}{\gamma_{r 1} P_{1} \gamma_{2 r}\left(\bar{P}-P_{2}\right)+\gamma_{2 r}\left(\bar{P}-P_{2}\right)+\gamma_{r 2} P_{2}+\gamma_{r 1} P_{1}+1}\right) \\
& =\frac{1}{2} \log \left(1+P_{2} \gamma_{22}+\frac{\gamma_{r 2} \gamma_{2 r}}{1+\eta_{r 1}} \frac{\left(\bar{P}-P_{2}\right) P_{2}}{\gamma_{2 r}\left(\bar{P}-P_{2}\right)+\frac{\gamma_{r 2}}{1+\eta_{r 1}} P_{2}+1}\right)
\end{aligned}
$$

One can check that $R_{2}^{e q}\left(P_{2}\right)$ is concave in $P_{2}$. Indeed, let $g\left(P_{2}\right)=\frac{\left(\bar{P}-P_{2}\right) P_{2}}{\gamma_{2 r}\left(\bar{P}-P_{2}\right)+\frac{\gamma_{r 2}}{1+\eta_{r 1}} P_{2}+1}, b=\frac{\gamma_{r 2}}{1+\eta_{r 1}}$. The derivatives of the function $g$ are given by

$$
\begin{aligned}
\dot{g}\left(P_{2}\right) & =-\frac{\bar{P}-2 P_{2}+\gamma_{2 r} \bar{P}^{2}+\gamma_{2 r} P_{2}^{2}-b P_{2}^{2}-2 \bar{P} \gamma_{2 r} P_{2}}{\left(\bar{P} \gamma_{2 r}-\gamma_{2 r} P_{2}+b P_{2}+1\right)^{3}} \\
\ddot{g}\left(P_{2}\right) & =-\frac{2\left(\bar{P} \gamma_{2 r}+1\right)(\bar{P} b+1)}{\left(\bar{P} \gamma_{2 r}-\gamma_{2 r} P_{2}+b P_{2}+1\right)^{3}} .
\end{aligned}
$$

Clearly, $\ddot{g}\left(P_{2}\right) \leq 0$ for all $P_{2} \in[0, \bar{P}]$. Therefore, $\mathrm{g}$ is concave in $P_{2}$ and hence $R_{2}^{e q}\left(P_{2}\right)$ is also concave in $P_{2}$. Thus, to solve (30), we first solve $\frac{d R_{2}^{e q}}{d P_{2}}\left(P_{2}\right)=0$ or equivalently we solve the second order polynomial equation given by $\gamma_{22}+$ $\gamma_{2 r} b \dot{g}\left(P_{2}\right)=0$. If the last equation possesses a solution in $\left[0, \min \left(\bar{P}, P_{\text {out }, 1}^{-1}(\epsilon)\right)\right]$, then it will be the optimal solution to (30). Otherwise, $P_{2}^{\text {opt }}=\min \left(\bar{P}, P_{\text {out }, 1}^{-1}(\epsilon)\right)$.

Example 4. We consider the setup given by $\bar{P}=10 \mathrm{~dB}, P_{1}=$ $10 \mathrm{~dB}$, and $R_{t}=1 \mathrm{bit} / \mathrm{s} / \mathrm{Hz}$. We consider Rayleigh-fading such that $h_{i i} \sim \mathcal{C N}(0,1), i \in\{1,2\}$ and $h_{i j} \sim \mathcal{C N}(0,3), i \neq j$. For each channels' realization, we compute the optimal power allocation solution of (29) and then we average over $10^{5}$ realizations. For comparison, for each channel link parameters, we consider the situation in which the relay power is fixed and set to $P_{r}=5 \mathrm{~dB}$. We plot the optimal rate $R_{2}$ under this setup as the solution to (25) with the same power budget, i.e., $\bar{P}_{2}=\bar{P}-P_{r}=5 \mathrm{~dB}$. We plot the result in Fig. 6 for different values of the outage constraint. Figure 6 shows the 

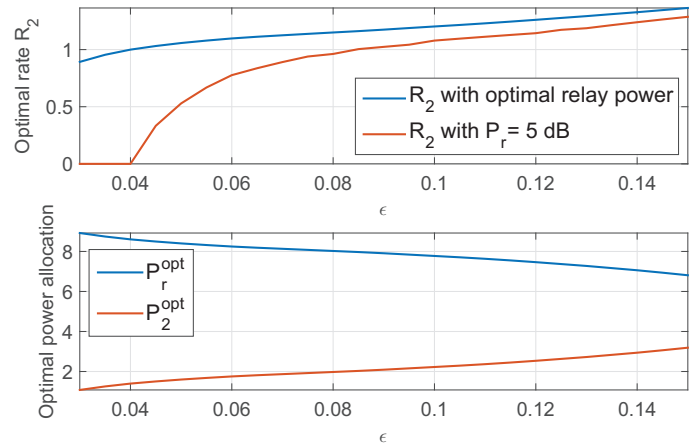

Fig. 6. Average achievable Rate of User 2 with optimal relay power allocation

benefit of optimizing jointly over the relay and the secondary user transmission power. This allows the secondary user to achieve a positive transmission rate while satisfying a lower outage values that are not achievable initially with fixed $P_{r}$ (all $\epsilon \leq 0.04$ in our example). Figure 6 shows also the optimal power strategy, on average. As the outage constraint is relaxed, i.e., $\epsilon$ increases, we allocate less and less power to the relay and more and more power to the secondary transmitter.

\section{CONCLUSION}

In this paper, we considered a two-user interference channel assisted by a relay. We have derived achievable rates of both users, by considering the interference as noise. we have solved the problem of maximizing the weighted sum rate of the system with and without relay power optimization. In particular, we proposed a simple algorithm solving the latter case. Then, we have solved the problem of maximizing the secondary user achievable rate subject to an outage constraint with respect to the primary user and a peak power constraint. We showed that jointly optimizing over the secondary user and relay transmit powers increases the secondary user rate.

\section{APPENDIX A}

To compute (26), we need to compute the distribution (precisely the cumulative distribution function (CDF) ) of $\frac{\eta_{1 r} \eta_{r 1} /\left(1+\eta_{r 2}\right)}{\eta_{1 r}+1+\eta_{r 1} /\left(1+\eta_{r 2}\right)}$ conditioned on $\eta_{r 1}, \eta_{r 2}$, as well as the distribution of $\eta_{11}$. Essentially, we need the CDF of the random variable $h=\frac{\gamma}{\gamma+c}$ where $c$ is a positive constant. This is expressed in the following lemma.

Lemma 2. Let $\gamma$ be a random variable with known $\operatorname{CDF} F_{\gamma}(\cdot)$, then, for a given positive constant $c$, the CDF of the random variable $h=\frac{\gamma}{\gamma+c}$, denoted $F_{h}(\cdot)$, is given by

$$
F_{h}(x)= \begin{cases}0 & \text { if } x \leq 0 \\ F_{\gamma}\left(\frac{c x}{1-x}\right) & \text { if } 0 \leq x<1 \\ 1 & \text { if } x \geq 1\end{cases}
$$

Proof: The proof is immediate and thus omitted.

Let $c=1+\eta_{r 1} /\left(1+\eta_{r 2}\right)$. Using the total probability theorem, we obtain

$$
\begin{aligned}
& P_{\text {out }, 1} \\
& =\int_{\eta_{11}} \operatorname{Pr}\left(\frac{\eta_{1 r} \eta_{r 1} /\left(1+\eta_{r 2}\right)}{\eta_{1 r}+1+\eta_{r 1} /\left(1+\eta_{r 2}\right)} \leq 2^{2 R_{t}}-1-\eta_{11}\right) f_{\eta_{11}}\left(\eta_{11}\right) d \eta_{11} \\
& =\int_{\eta_{11}} \operatorname{Pr}\left(\frac{\eta_{1 r}}{\eta_{1 r}+c} \leq \frac{2^{2 R_{t}}-1-\eta_{11}}{c-1}\right) f_{\eta_{11}}\left(\eta_{11}\right) d \eta_{11} .
\end{aligned}
$$

Using Lemma 2, (35) can be computed as

$$
\begin{aligned}
& P_{\text {out }, 1} \\
& =\int_{\left[2^{2 R_{t}}-c\right]^{+}}^{2^{2 R_{t}}-1} \operatorname{Pr}\left(\frac{\eta_{1 r}}{\eta_{1 r}+c} \leq \frac{2^{2 R_{t}}-1-\eta_{11}}{c-1}\right) f_{\eta_{11}}\left(\eta_{11}\right) d \eta_{11} \\
& +\int_{0}^{\left[2^{2 R_{t}}-c\right]^{+}} f_{\eta_{11}}\left(\eta_{11}\right) d \eta_{11} \\
& =\int_{\left[2^{2 R_{t}}-c\right]^{+}}^{2^{2 R_{t}}-1} F_{\frac{\eta_{1 r}}{\eta_{1 r}+c}}\left(\frac{2^{2 R_{t}}-1-\eta_{11}}{c-1}\right) f_{\eta_{11}}\left(\eta_{11}\right) d \eta_{11} \\
& +\int_{0}^{\left[2^{\left.2 R_{t}-c\right]^{+}}\right.} f_{\eta_{11}}\left(\eta_{11}\right) d \eta_{11} \\
& =\int_{\left[2^{\left.2 R_{t}-c\right]^{+}}\right.}^{2^{2 R_{t}}-1} F_{\eta_{1 r}}\left(\frac{c x}{1-x}\right) f_{\eta_{11}}\left(\eta_{11}\right) d \eta_{11} \\
& +\int_{0}^{\left[2^{2 R_{t}}-c\right]^{+}} f_{\eta_{11}}\left(\eta_{11}\right) d \eta_{11} \\
& =\int_{\left[2^{2 R_{t}}-c\right]^{+}}^{2^{2 R_{t}}-1} F_{\gamma_{1 r}}\left(\frac{c x}{P_{r}(1-x)}\right) f_{\eta_{11}}\left(\eta_{11}\right) d \eta_{11} \\
& +\int_{0}^{\left[2^{2 R_{t}}-c\right]^{+}} f_{\eta_{11}}\left(\eta_{11}\right) d \eta_{11}
\end{aligned}
$$

where $f_{\eta_{11}}\left(\eta_{11}\right)=\frac{f_{\gamma_{11}}\left(\frac{\eta_{11}}{P_{1}}\right)}{P_{1}}$ and $x=\frac{2^{2 R_{t}}-1-\eta_{11}}{c-1}$.

\section{REFERENCES}

[1] T. Cover and A. Gamal, "Capacity theorems for the relay channel," IEEE Trans. Inf. Theory, vol. 25, no. 5, pp. 572-584, Sep 1979.

[2] A. Sendonaris, E. Erkip, and B. Aazhang, "User cooperation diversity. part i. system description," IEEE Trans. Commun, vol. 51, no. 11, pp. 1927-1938, Nov 2003.

[3] A. Bletsas, A. Khisti, D. Reed, and A. Lippman, "A simple cooperative diversity method based on network path selection," IEEE J. Sel. Areas Commun, vol. 24, no. 3, pp. 659-672, March 2006.

[4] Y. Jing and H. Jafarkhani, "Single and multiple relay selection schemes and their achievable diversity orders," IEEE Trans. Wireless Commun, vol. 8, no. 3, pp. 1414-1423, March 2009.

[5] Y. Yao, X. Cai, and G. Giannakis, "On energy efficiency and optimum resource allocation of relay transmissions in the low-power regime," IEEE Trans. Wireless Commun, vol. 4, no. 6, pp. 2917-2927, Nov 2005.

[6] A. Host-Madsen and J. Zhang, "Capacity bounds and power allocation for wireless relay channels," IEEE Trans. Inf. Theory, vol. 51, no. 6, pp. 2020-2040, June 2005.

[7] X. Deng and A. Haimovich, "Power allocation for cooperative relaying in wireless networks," IEEE Commun. Lett, vol. 9, no. 11, pp. 994-996, Nov 2005.

[8] O. Sahin, E. Erkip, and O. Simeone, "Interference channel with a relay: Models, relaying strategies, bounds," in Information Theory and Applications Workshop, 2009, Feb 2009, pp. 90-95.

[9] I. Maric, R. Dabora, and A. Goldsmith, "An outer bound for the gaussian interference channel with a relay," in Information Theory Workshop, 2009. ITW 2009. IEEE, Oct 2009, pp. 569-573.

[10] S. Rini, D. Tuninetti, N. Devroye, and A. Goldsmith, "On the capacity of the interference channel with a cognitive relay," IEEE Trans. Inf. Theory, vol. 60, no. 4, pp. 2148-2179, April 2014.

[11] M. Costa and A. Gamal, "The capacity region of the discrete memoryless interference channel with strong interference (corresp.)," IEEE Trans. Inf. Theory, vol. 33, no. 5, pp. 710-711, Sep 1987.

[12] I. Maric, R. Dabora, and A. Goldsmith, "Relaying in the presence of interference: Achievable rates, interference forwarding, and outer bounds," IEEE Trans. Inf. Theory, vol. 58, no. 7, pp. 4342-4354, July 2012.

[13] F. Reategui, M. Imran, and R. Tafazolli, "On the capacity of the cognitive interference channel with a relay," in Wireless Communications and Networking Conference (WCNC), 2014 IEEE, April 2014, pp. 149-153.

[14] S. ping Yeh and O. Leveque, "Asymptotic capacity of multi-level amplify-and-forward relay networks," in IEEE International Symposium on Information Theory, 2007. ISIT 2007., June 2007, pp. 1436-1440.

[15] K. Cumanan, R. Zhang, and S. Lambotharan, "A new design paradigm for MIMO cognitive radio with primary user rate constraint," IEEE Commun. Lett, vol. 16, no. 5, pp. 706-709, 2012.

[16] T. M. Cover and J. A. Thomas, Elements of information theory. John Wiley \& Sons, 2012. 